\title{
DENOISING OF HEART SOUND SIGNAL USING WAVELET TRANSFORM
}

\author{
Gyanaprava Mishra $^{1}$, Kumar Biswal ${ }^{2}$, Asit Kumar Mishra ${ }^{3}$ \\ ${ }^{1,2}$ Department of Electronics \& Instrumentation Engineering, ITER/SOA University, India \\ ${ }^{3}$ Department of Electronics \& Telecommunication Engineering, GDRCET/CSVT University, India \\ gyanaprava.mishra022@gmail.com
}

\begin{abstract}
This paper presents a novel wavelet-based denoising method using coefficient thresholding technique. The proposed method uses the adaptive thresholding which overcome the shortcomings of discontinuous function in hard-thresholding and also can eliminate the permanent bias in soft-thresholding. The qualitative evaluation of the denoising performance has shown that the proposed method cancels noises more effectively than the other examined techniques. The introduced method can be used as preprocessor stage in all fields of phonocardiography, including the recording of fetal heart sounds on the maternal abdominal surface.
\end{abstract}

Keywords - PCG signal, wavelets, signals to noise ratio (SNR), percentage of reconstruction (PR).

\section{INTRODUCTION}

Phonocardiography is one of the best graphical representations of the heart sound and murmurs, which documents the timing and annotates their different relative intensities and provides valuable information concerning the heart valves and hemodynamics. Unfortunately the heart sound signal is very weak and can be easily subject to interference from various noise sources. These various noise components make the diagnostic evaluation of phono cardio graphic (PCG) records difficult or in some cases even impossible.

Cardiovascular diseases are the 21st century epidemic. Ageing, obesity, sedentary lifestyle and numerous other factors contribute to its growing numbers, with devastating causes, both economic and social. Heart sound provides clinicians with valuable diagnostic and prognostic information. Cardiac auscultation is one of the oldest methods for heart function assessment as it is a non invasive, low cost method which provides accurate information about heart mechanics and hemodynamics [1], [2], [7], [8].

Unfortunately, heart sound recordings are very often disturbed by various factors such as: respiration sounds (lung mechanics), patient movements, small movements of the stethoscope ("shear noises"), acoustic damping through the bones and tissues, and external noises from the environment etc. In case of fetal phonocardiography the most commons disturbances are: acoustic damping of forewaters and maternal tissues, acoustic noises produced by the fetal movements, noises of the maternal digestive system, and sounds of maternal heart.
Most of the existing phonocardiographic processing methods concern only with the diagnostic analysis of heart sounds without an adequate emphasis on the denoising of the PCG records. Existing methods usually apply digital band-pass filters (most commonly IIR-filters of FFT-based filtering) as a simple denoising method. The cut-off frequencies of the filters are determined by empirical observations, and commonly the pass band lies between 30 and $200 \mathrm{~Hz}$ [3], [4], [5], [6], [7].

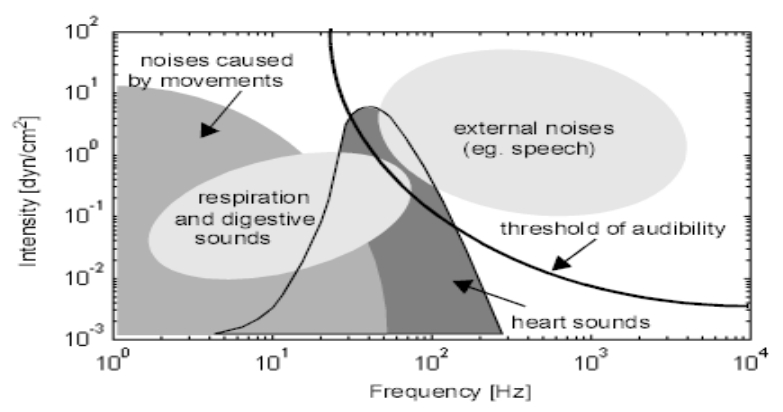

Fig. 1: Spectral intensity map of PCG records [8].

This paper presents a novel technique, which allows a more effective noise cancellation, also can be used as an advanced preprocessing stage in phonocardiographic diagnosis analyzer systems.

\section{METHODS}

In this proposed method Wavelet Transform is used for denoising of PCG signal, as wavelet allows to do multiresolution analysis, which helps to achieve both time and frequency localization. Wavelet algorithms process the data at different scales or resolutions. In this proposed method we have studied the performance of different wavelets on PCG 
signal and an attempt is made to find the wavelet which gives the higher result for signal to noise ratio for all types of PCG signal and best level of reconstruction.

The coefficients of the Wavelet Transform (WT) of any signal contain important information whose amplitude is large, while wavelet coefficients of noise are small in amplitude. Selecting an appropriate threshold in different scale, the coefficients will be set to zero if it is below the threshold, while be retained if above the threshold, so that the noise in the signal is effectively suppressed. [9], [10]. Finally the reconstructed and filtered signals are obtained using wavelet inverse transform [11].

A wavelet is simply a small wave which has energy concentrated in very small time duration, of which the main lobe contains approximately the $98 \%$ of the energy and the side lobes contains the rest $2 \%$ of the energy given in equation (1) and depicted in figure 2. The wavelet basis's shifting and translation capability enables the wavelet to equip with flexible and variable time and frequency windows that narrow down at high frequencies and broaden at low frequencies, making it available to localize on any detail of the analytical object. Hence due to these enormous properties Wavelet Transform is suitable for analyzing such a highly unstable, transient, nonstationary signal like phonocardiogram (PCG). heart sound signals. As a result, the multi-resolution analysis of the wavelet has good characteristics and advantages in both the space domain and frequency domain [12].

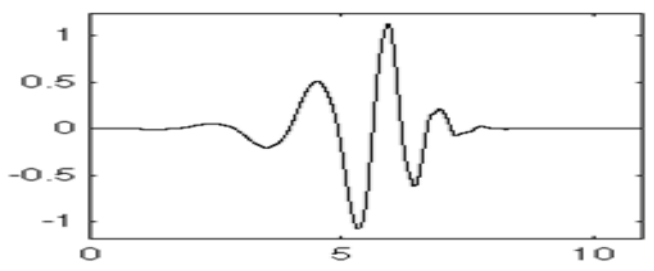

Fig: 2 db6 wavelet

$$
\mathrm{CWT}_{\mathrm{f}(\mathrm{t})}^{\Psi}=\Psi_{\mathrm{f}(\mathrm{t})}^{\Psi}=\frac{1}{\sqrt{|\mathrm{s}|}} \int \mathrm{f}(\mathrm{t}) \Psi^{*}\left(\frac{\mathrm{t}-\tau}{\mathrm{s}}\right) \mathrm{dt} \ldots(1)
$$

A signal $\mathrm{f}(\mathrm{t})$ can be better analyzed and expressed as a linear decomposition of the sums or products of the coefficient and function of a wavelet function shown in Fig. 2. The set of coefficients are called the Wavelet Transform of $f(t)$, which maps the function $f(t)$ of a continuous variable into a sequence of coefficients having four properties. The representation of singularities, the representation of local basis functions to make the algorithms adaptive in-homogeneities of the functions, also having the unconditional basis property for a variety of function classes to provide a wide range of information.

\section{A. THE BASIC ONE DIMENSIONAL MODEL}

The noisy signal is obtained by generating and adding a white Gaussian noise to the original signal, mathematically given by

$$
\mathrm{V}_{\mathrm{s}}(\mathrm{n})=\mathrm{V}(\mathrm{n})+\mathrm{S}(\mathrm{n})
$$

Where S (n) is the white Gaussian noise, V (n) noiseless PCG signal without noise and Vs (n) is the noisy PCG signal. Figure 3 depicts the plot of noiseless and noisy PCG signal.
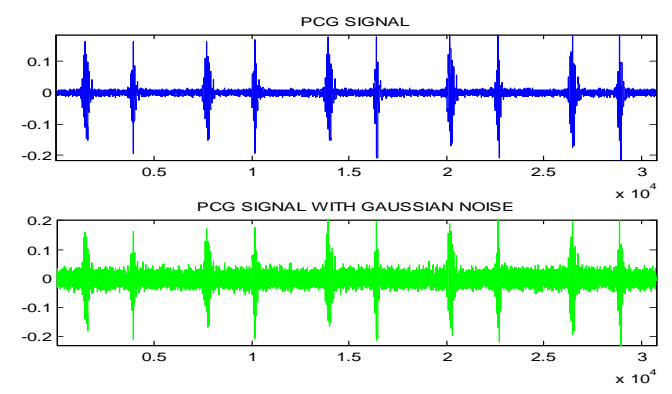

Fig. 3: PCG Signal without and with Noise

\section{B. DENOISING PROCEDURES}

The de-noising objective is to suppress the noise part of the signal S (n) and to recover V (n). From a statistical viewpoint, the model is a regression model over time and the method can be viewed as a nonparametric estimation of the function $\mathrm{V}(\mathrm{n})$ using orthogonal basis. The de-noising procedures are followed in three steps:

\section{Decompose:}

Choose a wavelet; choose a level L. Compute the wavelet decomposition of the signal $\mathrm{s}$ at level $\mathrm{L}$.

\section{Threshold detail coefficients:}

Then this transforms (decomposed wavelet coefficients) are passed through a threshold, which removes the coefficients below a certain value For each level from 1 to $\mathrm{L}$, select a threshold and apply the adaptive thresholding to the detail coefficients, given by

$$
\mathrm{T}=\sqrt{|\mathrm{M}(\mathrm{n})+\sigma(\mathrm{n})|}
$$

Where $M(n)$ : mean of the $n$ wavelet coefficients and $\sigma(n)$ : standard deviation of the $\mathrm{n}$ wavelet coefficient.

\section{Choosing and applying threshold value:}

This paper suggests an adaptive thresholding method which decides the different thresholding value at different level of decomposition for various Wavelets. For each level a threshold value is found through a loop, and it is applied for the detailed coefficients of the noisy and original signals. The optimum threshold is chosen by taking the minimum error between the detailed coefficients of noisy signal and those for original 
signal. A soft thresholding is used to shrinkage the wavelet detailed coefficients of the noisy signal such that:

$$
\mathrm{C}_{\mathrm{o}}(\mathrm{s}, \tau)\left\{\begin{array}{ll}
=\mathrm{C}(\mathrm{s}, \tau) & \text { if } \mathrm{C}(\mathrm{s}, \tau) \geq \mathrm{T} \\
=0 & \text { if } \mathrm{C}(\mathrm{s}, \tau) \leq \mathrm{T}
\end{array}\right\}
$$

Where $\mathrm{C}(\mathrm{s}, \tau)$ : wavelet transform coefficients, $\mathrm{Co}(\mathrm{s}, \tau)$ : is the output wavelet transform coefficients after thresholding, and $\mathrm{T}$ is the chosen threshold. Threshold determination using above method and the idea of not to threshold the approximation coefficients of PCG signal. The approximation coefficients contain the low frequency of the original signal where most energy exists.

\section{Reconstruction:}

The original signal is reconstructed using Inverse Wavelet Transform IDWR (Fig.4).Thresholding of wavelet coefficients affects greatly the quality of PCG morphology, thus, threshold determination is very essential issue in this case.

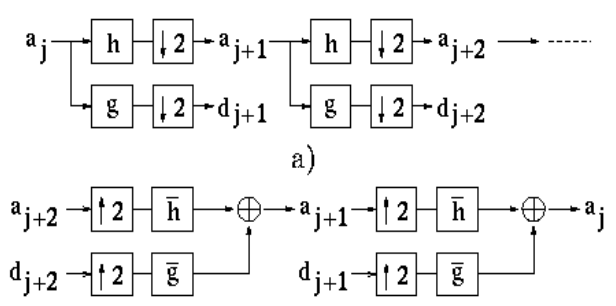

b)

Fig 4 (a) decomposition, (b) reconstruction

Two parameters are used to ensure the qualitative studies of PCG denoising are:

\section{Signal to Noise Ratio (SNR):}

Signal-to-noise-ratio is a traditional method of measuring the amount of noise present in a signal. The standard definition of the SNR is the following, considering both signals $\mathrm{V}(\mathrm{n})$ and noise $\mathrm{S}(\mathrm{n})$ individually, during respective time periods $\mathrm{L}$ and $\mathrm{N}$ :

The SNR is given by:

$$
\begin{aligned}
(\mathrm{SNR} & =10 * \log \left(\text { Power }_{\text {signal }} / \text { Power }_{\text {noise }}\right) \\
& =\frac{\frac{1}{\mathrm{~L}} \sum_{\mathrm{i}=1}^{\mathrm{L}} \mathrm{x}_{\mathrm{i}}^{2}}{\frac{1}{\mathrm{~N}} \sum_{\mathrm{i}=1}^{\mathrm{N}} \mathrm{n}_{\mathrm{i}}^{2}}(\text { in } \mathrm{dB}) \quad \ldots . .(4.4)
\end{aligned}
$$

\section{Percentage of Signal Reconstruction:}

The reconstruction level is analysed by the factor percentage of reconstruction given by

$$
\mathrm{PR}=(1-\varepsilon) * 100
$$

Error signal given by

$$
\varepsilon=\frac{S(n)-S_{R}(n)}{S_{R}(n)}
$$

Where S (n): original PCG signal and SR (n): reconstructed PCG signal.

\section{RESULTS AND DISCUSSION}

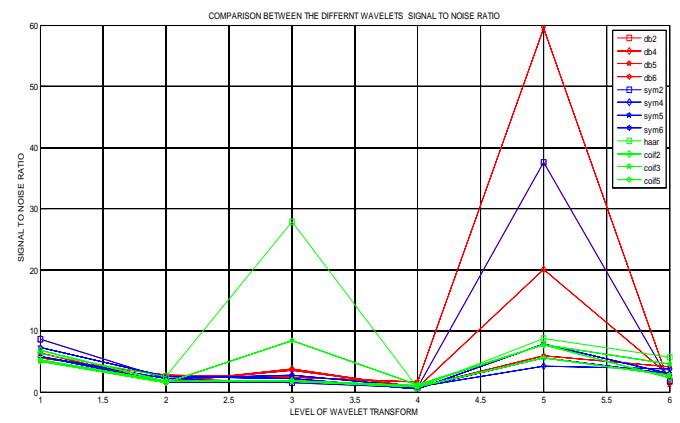

Fig 5: Plot of SNR at various level for different wavelet

The noisy PCG signal is tested with twelve different types of wavelet functions at six different levels. The result is depicted bellow in figure 5 which shows that, the wavelet function daubechies 5 (db5) gives maximum SNR at 5 level of decomposition giving the percentage of reconstruction moderate value. While, $\mathrm{db} 5$ at level 2 producing the maximum percentage of reconstruction given in table 1 . The denoising results of denoising at the six different level of decomposition for db5 using the above relationship depicted in Fig. 6

Table 1 Percentage of Reconstruction

\begin{tabular}{|c|c|c|c|c|c|c|}
\hline $\begin{array}{c}\text { Wavelet } \\
\text { Name / } \\
\text { Level }\end{array}$ & 1 & 2 & 3 & 4 & 5 & 6 \\
\hline $\mathrm{db} 2$ & 88.46 & 84.88 & 79.5 & 80.91 & 91.11 & 45.92 \\
\hline $\mathrm{db} 4$ & 93.67 & 92.73 & 91.42 & 75.57 & 65.89 & 66.69 \\
\hline $\mathrm{db} 5$ & 91.28 & $\mathbf{9 8 . 1 9}$ & 77.77 & 83.19 & $\mathbf{9 2 . 0 6}$ & 82.63 \\
\hline $\mathrm{db} 6$ & 88.06 & 91.83 & 83.31 & 73.23 & 89.9 & 57.02 \\
\hline sym2 & 88.46 & 84.88 & 79.5 & 80.91 & 91.11 & 45.92 \\
\hline $\mathrm{sym} 4$ & 89.4 & 89.44 & 81.29 & 79.11 & 66.56 & 56.19 \\
\hline $\mathrm{sym5}$ & 87.84 & 91.05 & 91.19 & 89.64 & 70.15 & 77.57 \\
\hline
\end{tabular}




\begin{tabular}{|c|c|c|c|c|c|c|} 
sym6 & 89.21 & 93.51 & 91.21 & 90.13 & 67.79 & 87.12 \\
\hline haar & 88.80 & 92.22 & 76.71 & 71.18 & 60.04 & 54.32 \\
\hline coif2 & 92.44 & 81.28 & 78.79 & 84.85 & 91.47 & 77.14 \\
\hline coif3 & 87.98 & 88.49 & 93.04 & 74.57 & 89.78 & 72.13 \\
\hline coif5 & 88.29 & 87.07 & 89.7 & 76.17 & 90.38 & 62.52 \\
\hline
\end{tabular}

For the future we design a wavelet which gives the much better result for the PCG signal. Also suggests some methods for denoising of PCG signals affected by different diseases. It is very much helpful for the physician to analysis the heart disease as easy and accurate.

\section{ACKNOWLEDGEMENTS}

The authors would like to thank Prof. Arun Kumar for his contribution to prepare this paper. Also the author would like to thank the E\&I department ITER for the whole hearted support. Furthermore, the authors would like to acknowledge the anonymous reviewers for their fruitful and constructive comments. We give our sincere thanks to internet sites [13] for providing the different heart sounds.

\section{REFERENCES}

[1] D. W. Sapire, "Understanding and diagnosing pediatric heart disease: Heart sounds and murmurs," Appleton \& Lange, Norwalk, Connecticut, pp. 27-43., 1992.

[2] G. S. Dawes, M. Moulden, C. W. Redman, "Imporvements in computerized fetal heart rate analysis antepartum," J. Perinatal Medicine, vol. 24, pp. 25-36., 1996.

[3] J. C. Wood, D. T. Barry, "Time-Frequency Analysis of the First Heart Sound," IEEE EMBS Magazine, pp. 144-151., March-April 1995.

[4] J. M. Bentley, P. M. Grant, J. T. E. McDonnel, "TimeFrequency and Time-Scale Techniques for the Classification of Native and Bioprosthetic Heart Valve Sounds," IEEE Trans. Biomedical. Engg., vol. 45, pp. 125-128., January 1998.

[5] J. Ritola, S. Lukkarinen, "Comparison of Time- Frequency Distributions in the Heart Sounds Analysis," Medical \& Biological Engineering \& Computing, vol 34., supplement 1., part 1., pp. 89-90., 1996.

[6] H. Liang, S. Lukkarinen, I. Hartimo, "Heart Sound Segmentation Algorithm Based on Heart Sound Envelogram," in Proc. Computers in Cardiology, vol. 24., 1997.

[7] F. Kovács, M. Török, I. Habermajer, "A Rule-Based Phonocardiographic Method for Long-Term Fetal Heart Rate Monitoring," IEEE Trans. Biomed. Engg., vol. 47., pp. 124130., January 2000.

[8] P. Várady, I. Gross, A. Hein, L. Chouk, "Analysis of the Fetal Heart Activity by the Means of Phonocardiography," Proc. IFAC Int. Conf. on Telematics and Automation, TA2001, Weingarten, Germany, July 2001.

[9] Vikhe P.S., Hamde S.T. and Nehe N.S. (2009) International Conference on Advances in Computing, Control, and Telecommunication Technologies, 367-371.

[10] Donoho L. (1995) IEEE Transactions. 41(3) 613-627.

[11] Rangaraj M. Rangayyan (2004) John Wiley \& Sons, 278280.

[12] Micheal Unser (1996) 8th IEEE Signal Processing Workshop, 244-249.

[13] [Online] Available: http://www.medicalstudent.com and physionet.com 


\section{BIOGRAPHIES:}

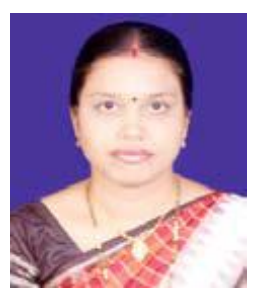

Gyanaprava Mishra: She received her B.E. Degree in Electronics \& Instrumentation from BPUT, Odisha. M.Tech scholar of Electronics \& Instrumentation with Specialization in VLSI \& Embedded System from SOA University, Odisha.

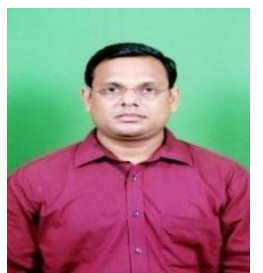

Kumar Biswal: He Received his B.Tech. Degree in Instrumentation \& Electronics from College of Engineering \& Technology (C.E.T), O.U.A.T University, Odisha, and M.Tech in Electrical Engineering with Specialization in Instrumentation from IIT Kharagpur,West Bengal. He is presently, working as Senior Asst. Prof. in the department of Electronics \&Instrumentation Engg.

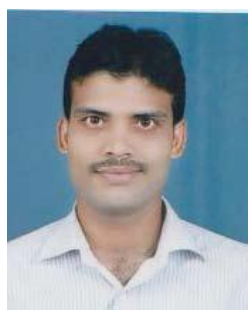

Asit Kumar Mishra: He Received his B.E. Degree in Electronics \& Instrumentation Engineering from NIST, Berhampur University, Berhampur, Odisha and MTech in Electronics and Telecommunication with specialization in Instrumentation and Control from CSVT University, Durg, Chhattishgarh, India. He is presently, working as Reader in the Deptt. of Electronics \& Telecomm Engg., GDRCET, Bhilai. 